\title{
Client's Location Hiding in Geosocial Recommendation Applications
}

\author{
Khadke Shriram Bhanudasrao', Mahadik Pravin B. ${ }^{2}$ \\ ${ }^{1}$ Department of Computer Science and Engineering Marthawada Shikshan Prasark Mandal's Deogiri Institute of Engineering \& \\ Management Studies, Aurangabad Maharashtra state, India 2015-16 \\ ${ }^{2}$ Assistant Professor Department of Computer Science and Engineering Marthawada Shikshan Prasark Mandal's Deogiri Institute of \\ Engineering \& Management Studies, Aurangabad Maharashtra state, India 2015-16
}

\begin{abstract}
Customer's Location Hiding In FourSquare is one of the Geosocial application in which lots of communities interrelate with their surrounding surroundings through their friends and their recommendations. With respect to security issues Geosocial application can simply misused, for instance to follow the user or target them for home invasions. In this way giving the protection to the Geosocial application is the research issue; existing system provides location protection without including vulnerability into the question results or depending on strong assumptions about server security. In existing systems the user send message to the another user is of greater size, in this way the expenses of the server database is increases on existing system. In this manner in the proposed system which provides the security to the user's location as well as uses compression calculation to compress and decompress the message so that that the message recovery the reality of the situation will become obvious eventually lesser than the previous system . By using our proposed system cost of the server database will decrease and the time required for transmitting the message is also decreases. The system encrypts the message for the security purpose. The proposed system provides security and improves the execution of the Geosocial application. The proposed system also uses keyed tags and arbitrary tags which adjust the protection and execution of the system. The keyed tag provides strong protection and irregular tags give security and high effectiveness to the system.
\end{abstract}

Keywords: Mobile location-based services, security, privacy

\section{Introduction}

Geo-social networks (GeoSNs) give a setting mindful administration that partners location with clients and content. The multiplication of GeoSNs shows that they're quickly pulling in clients. GeoSNs right now offer diverse sorts of administrations, with photo sharing, friend tracking, and "check-ins." However, this capacity to uncover clients locations causes new privacy dangers, which thus call for new privacy-security techniques. The creators study four privacy angles key to these social networks - location, nonappearance, co-location, and personality privacy - and portray conceivable method for securing privacy in these circumstances. In today's reality, Smartphone applications have gotten to be prominent among the clients improving figuring stage. A sort of use is coming into line light that can be put under the class of geosocial application. Samples of this social application are nearby friend proposal for eating and shopping, and additionally amusements and cooperative system administrations. Yet, it has been seen that these application demonstrate detriments as there is a danger of losing clients privacy, at present because of negligible privacy instrument. Client's all think about the "spots" highlight of facebook which was abused by a few hoodlums. Subsequently, there is a genuine requirement for more grounded privacy properties with a specific end goal to make it all the more friendly to the clients.

Presently, Geosocial application have turned out to be an integral part of human lives. Be that as it may, these might be abused by somebody to concentrate clients close to home data. LocX has a tendency to give enhanced privacy and with result very certain. The essential thing that is done is to utilize secure direction change. This change would be utilized just by friends of a specific client. It permits the server to work legitimately and accurately without getting to the private information of the client. There are clients where there is not a requirement for subjective sets of clients to be determined. Subsequently, by recognizing such location information through clients social gatherings and further change can be utilized on location coordination. The direction changes protect separation measurements, improving the errand of server to perform inquiries on changed information. The change is a protected one, since the key is mystery which knows just to the clients bunch.

The proposed framework utilizes the compression strategy. LZW compression calculation is utilized for compression. LZW compression is quick and easy to apply. Since this is a lossless compression strategy, none of the substance in the record are lost amid or after compression. Sender first sends GPS location. Like the LocX procedure use changes the coordinates and spare those on to the list disjoin .The compression strategy is utilized the pack the document and then apply the encryption. This procedure has points of interest of having the capacity to send extensive records to the cell phones which has less memory than the normal PCs The framework in which the compression system is utilized while client send the message to the another us size so the er at first client scramble the message by the encryption calculation and after that pack the message and send to another client. Moreover client includes key hash and random hash labels for enhancing the privacy and execution of the framework. Key hash is essentially more proficient than no labels as far as handling time on the client's gadget, while giving the same, solid privacy. The random hash gives both high privacy and high proficiency.

\section{Volume 5 Issue 2, February 2016}




\section{International Journal of Science and Research (IJSR) \\ ISSN (Online): 2319-7064}

Index Copernicus Value (2013): 6.14 | Impact Factor (2014): 5.611

\section{Literature Survey}

B. Gedik and L. Liu depicts [4] a customized k-anonymity model for ensuring location privacy against different privacy dangers through location data sharing. Model has two novel elements. To begin with, it gives a bound together privacy personalization framework to bolster location k-anonymity for an extensive variety of clients with setting touchy customized privacy prerequisites. This framework empowers every versatile hub to indicate the base level of spatial resolutions it is willing to endure when asking for kanonymity protecting location-based services (LBSs). Second, it devises an effective message irritation motor which keeps running by the location assurance broker on a trusted server and performs location anonymization on portable clients' LBS ask for messages, for example, personality evacuation and spatio-worldly cloaking of location data.

P. Kalnis, G .Ghinita, K. Mouratidis, and D. Papadias [7] created routines for securing the privacy of clients issuing spatial inquiries against location-based attacks. In particular, keep an attacker from adapting so as to construing the personality of the question source the settled K-anonymity method to the spatial space. At the point when the client needs to discover some data utilizing location based administration (LBS) without revealing his data. The client utilizes an anonymizer, a trusted server .He sets up the protected association with anonymizer. Anonymizer uproots the ID of the client and changes his location through a system called cloaking. Cloaking shrouds the real location by a Kanonymizing spatial region (K-ASR or ASR), which is a range that encases the customer that issued the inquiry, and at any rate $\mathrm{K}-1$ different clients. The anonymizer then sends the ASR to the LBS, which comes back to the anonymizer an arrangement of candidate results that fulfill the inquiry condition for any conceivable point in the ASR. Asking the same question from progressive locations might unveil the personality of the questioning client, who will be incorporated into all ASRs.

T. Jiang, H.J. Wang, and Y.- C.Hu[6] states the three wellsprings of leakage of data in a wireless networks are characterized as time, location and sender hub personality. Framework investigates the accomplished location privacy of a portable hub utilizing the metric of privacy entropy. To muddle the transmission time, framework presents the deft noiseless period, which takes place amid the unmoving time between client's correspondence sessions. To keep an attacker from utilizing client personality for tracking, clients must utilize much of the time changing pseudonyms for interchanges. To keep an attacker from utilizing client personality for tracking, clients must utilize every now and again changing pseudonyms for interchanges. In a 802.11 WLAN, MAC and IP locations are client characters that should be ensured by utilizing pseudonyms. This privacyempowered frameworks penance administration quality. Clients in privacy mode will have their interchanges postponed in the event that they impart before a quiet period closes.
G. Ghinita, P. Kalnis, and S. Skiadopoulo[8] proposes Prive, an appropriated structural engineering for anonymous location-based questions, which addresses the issues of existing frameworks. (i) Develop a predominant K-ASR development system that ensures question anonymity regardless of the possibility that the attacker knows the locations of all clients. (ii) Introduce a disseminated convention utilized by versatile elements to self-compose into a deficiency tolerant overlay network. In Prive, K-ASRs are implicit a decentralized style, along these lines the bottleneck of the brought together server is kept away from. Following the condition of the framework is conveyed, Prive is strong to attacks. This methodology harms the precision and opportuneness of the reactions from the Server.

B.Hoh et al.[9] addresses the test of giving solid privacy ensures while keeping up high information precision of timearrangement location information. In particular, the key commitments of this work are:

1)Acquaintance of a novel time-with disarray metric to assess privacy in an arrangement of location follows.

2)Advancement of a vulnerability mindful privacy calculation that can promise a predetermined greatest timeto-perplexity.

S. Papadopoulos, S.Bakiras, and D.Papadias[15] proposes routines for subjective kNN look with solid location privacy. There are two fundamental segments in the proposed plan: (i) the PIR usefulness, and (ii) the question arrangement. The previous guarantees that the LBS is unaware of every block recovered by the calculations. Framework utilizes secure equipment PIR, which is the main down to earth decision for PIR in databases of non-negligible size. Specifically, this system offers private block recoveries with consistent correspondence cost and amortized poly-logarithmic computational expense. The question arrangement guarantees that each inquiry recovers the same number of blocks amid its execution. A trivial arrangement would authorize every inquiry to recover a settled and discretionarily expansive number of blocks. Its execution, albeit enhanced by utilizing unique equipment, however it is still much more awful than the various methodologies. In this way it is misty at present if this methodology can be connected in genuine LBSs.

\section{Proposed System}

In the framework the client who needs to share some data about any location recovers the co ordinates $(x, y)$ of that location from the GPS framework. At that point by utilizing the mystery pivot edge and move , he will change those coordinates say $\left(\mathrm{x}^{\prime}, \mathrm{y}^{\prime}\right)$. A random number generator is utilized to produce the list and is encoded with the mystery key .All the mystery data is passed on to the client's social using so as to gather some safe media like email or telephonic discussion.

At that point the changed co-ordinates alongside the scrambled record will be saved money on to the file server. The information relating to this location is encoded with the mystery key .This information is again compacted with the assistance of the compression calculation for the effective 


\section{International Journal of Science and Research (IJSR) \\ ISSN (Online): 2319-7064}

Index Copernicus Value (2013): 6.14 | Impact Factor (2014): 5.611

retrieval of the data from the information disjoin. There are different reviews present for the same location whether from client's social gathering or from the unknown clients, To recognize these two gatherings we can utilize the hash code. So the list server contains the one more field for the hash code which can be checked by the client 's friend to recover the genuine review from his social gathering. To determine the name clashes i.e same names for the better places the framework utilizes extraordinary labels. To enhance the execution of review retrieval from the information server we can utilize the compression component which packs the reviews and stores it to the information separate. At the point when client's friend needs to get to the reviews for the predetermined location again he changes the co-ordinates and sends the inquiry to the record server. At that point he recovers the file by utilizing secrete key .After recovering the file a different question will be terminated on to the information server to get the review. The review will initially decompressed and then decrypted with the same secrete key. Along these lines the proposals can be safely spoken with in the client 's social circle without presenting his location to the outside world

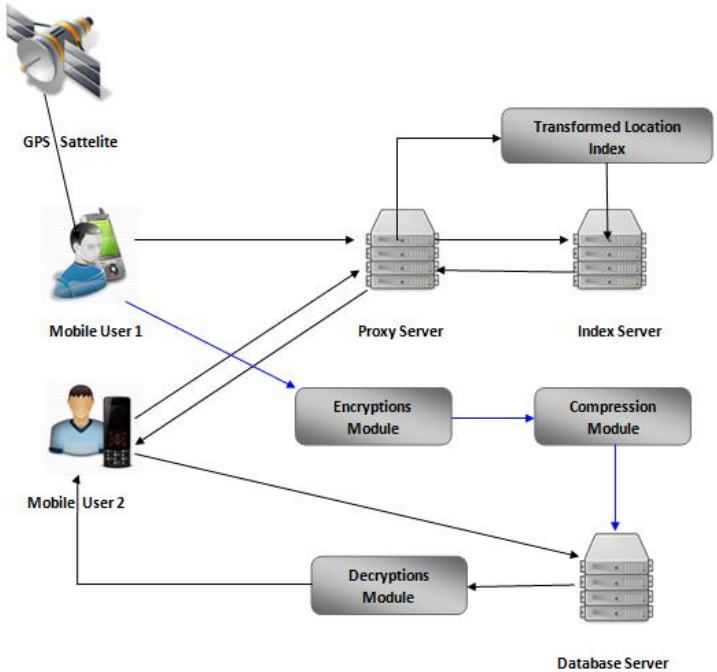

Figure 1: Overview of system operations

System uses following algorithms in our system:

1. Data Compression algorithm The user data stored in databases might generate bigger sized files. Thus, the Data Compression algorithm is used to compress the generated data. The user will get these compressed files from the database.

2. Data Decompression algorithm The files that user will get from the database will be in compressed form, as stated above. Therefore, a decompression algorithm is necessary.

3. AES Encryption algorithm We are using the AES Encryption algorithm, instead of any other, is because of the security that it provides. Here, the user location information will be encrypted before it is sent to the server for storage purpose. Therefore, even if the attacker gets this information somehow, it won't be able to access it.

4. AES Decryption algorithm The decryption algorithm is used to for decrypting the user location data, when the actual data will be necessary for the processing.

\section{Conclusion}

In this paper we examined about giving the security, privacy and expanding the execution of the location-based social network framework. Proposed work composed a security and privacy mindful convention for the framework and perceived its culmination and rightness. We utilized the compression system and seek by tag the procedure to build a for each formance of Geosocial application. Existing framework takes time for transmitting the data or data to the server and additionally the for getting the right information. It take more opportunity to transmit the message in light of the fact that the size of the message is huge, which corrupts the execution of the current framework. The proposed framework beats the drawback of the LocX framework and enhances the execution of the framework by utilizing the compression systems.

\section{References}

[1] B. Schilit, J. Hong, and M. Gruteser, Wireless Location Privacy Protection, Computer, vol. 36, no. 12, pp. 135137, Dec. 2003.

[2] M. Gruteser and D. Grunwald, Anonymous Usage of Location-Based Services through Spatial and Temporal Cloaking, Proc.First Intl Conf. Mobile Systems, Applications Services, 2003

[3] M. Motani, V. Srinivasan, and P.S. Nuggehalli, PeopleNet:Engineering a WirelessVirtual Social Network, Proc. ACM MobiCom, 2005

[4] B. Gedik and L. Liu, Location Privacy in Mobile Systems:A Personalized Anonymization Model, Proc. IEEE 25th Intl Conf.Distributed Computing Systems,2005.

[5] B. Hoh, M. Gruteser, H. Xiong, and A. Alrabady, Enhancing Security and Privacy in Traffic-Monitoring Systems, IEEE Pervasive Computing Magazine, vol. 5 , no. 4,pp. 38-46, Oct. 2006.

[6] T. Jiang, H.J. Wang, and Y.-C. Hu, Preserving Location Privacy in Wireless Lans, Proc. Fifth Intl Conf. Mobile Systems, Applications Services, 2007.

[7] P. Kalnis, G. Ghinita, K. Mouratidis, and D. Papadias, Preventing Location-Based Identity Inference in Anonymous Spatial Queries, IEEE Trans. Knowledge Data Eng., vol. 19, no. 12,pp. 1719-1733, Dec. 2007.

[8] G. Ghinita, P. Kalnis, and S. Skiadopoulos, PRIVE: Anonymous Location Based Queries in Distributed Mobile Systems, Proc. 16th Intl Conf. World Wide Web, 2007

[9] B. Hoh et al., Preserving Privacy in GPS Traces via Uncertainty Aware Path Cloaking, Proc. 14th ACM Conf. Computer Comm.Security, 2007.

[10]G. Ananthanarayanan, V.N. Padmanabhan, L. Ravindranath, and C.A. Thekkath,Combine: Leveraging the Power of Wireless Peers through Collaborative Downloading, Proc. Fifth Intl Conf.Mobile Systems, Applications Services, 2007.

[11]P. Mohan, V.N. Padmanabhan, and R. Ramjee, Nericell: Rich Monitoring of Road and Traffic Conditions Using Mobile Smartphones, Proc. Sixth ACM Conf. Embedded Network Sensor Systems, 2008 
[12] G. Ghinita, P. Kalnis, A. Khoshgozaran, C. Shahabi, and K.-L. Tan,Private Queries in Location Based Services: Anonymizers Are Not Necessary, Proc. ACM SIGMOD Intl Conf. Management Data,2008.

[13] J. Manweiler, R. Scudellari, and L.P. Cox, SMILE: Encounter Based Trust for Mobile Social Services, Proc. 16th ACM Conf.Computer Comm. Security (CCS 09), 2009.

[14] K.P.N. Puttaswamy, R. Bhagwan, and V.N. Padmanabhan,Anonygator: Anonymity and Integrity Preserving Data Aggregation, Proc. ACM/IFIP/USENIX 11th Intl Conf. Middleware(Middleware 10), 2010

[15] S. Papadopoulos, S. Bakiras, and D. Papadias, Nearest Neighbor Search with Strong Location Privacy, Proc. VLDB Endowment,vol. 3, nos. 1/2, pp. 619-629, Sept. 2010

[16] T. Isdal, M. Piatek, A. Krishnamurthy, and T. Anderson, Privacy Preserving P2P Data Sharing with Oneswarm, Proc. ACMSIG COMM, 2010.

[17]P. Gill et al., DudeWheres that IP? Circumventing Measurement Based IP Geolocation, Proc. 19th USENIX Conf. Security, p. 16,2010

[18] H. Hu, J. Xu, C. Ren, and B. Choi, Processing Private Queries over Untrusted Data Cloud through Privacy Homomorphism,Proc. IEEE 27th Intl Conf. Data Eng. (ICDE), 2011.

[19]A. Narayanan, N. Thiagarajan, M. Lakhani, M. Hamburg, and D. Boneh, Location User's Location Hiding In Geosocial Recommendation Applications. Privacy via Private Proximity Testing, Proc. Network Distributed System Security Conf., 2011.

[20] Krishna P. N. Puttaswamy, Shiyuan Wang, Troy Steinbauer, Divyakant Agrawal, Amr El Abbadi, Christopher Kruegel and Ben Y. Zhao, Preserving Location Privacy in Geo-Social Applications, IEEE transactions on mobile computing vol:13 no:1 year 2014 . 